Supporting information

\title{
Advanced porous membranes with tunable morphology regulated by ionic strength of nonsolvent for flow battery
}

\author{
Lin Qiao, ${ }^{+, \dagger}$ Huamin Zhang, ${ }^{+,}$Wenjing Lu, ${ }^{+}$Qing Dai ${ }^{+, \dagger}$ and Xianfeng Li ${ }^{*,+,}$
}

${ }^{+}$Division of Energy Storage, Dalian National Laboratory for Clean Energy, Dalian Institute of Chemical Physics, Chinese Academy of Sciences, Dalian 116023, China.

${ }^{\dagger}$ School of Chemistry and Chemical Engineering, University of Chinese Academy of Sciences, Beijing 100049, China.

${ }^{\S}$ Collaborative Innovation Centre of Chemistry for Energy Materials (iChEM), Dalian 116023, P. R. China.

*Corresponding Author. E-mail: lixianfeng@dicp.ac.cn. 


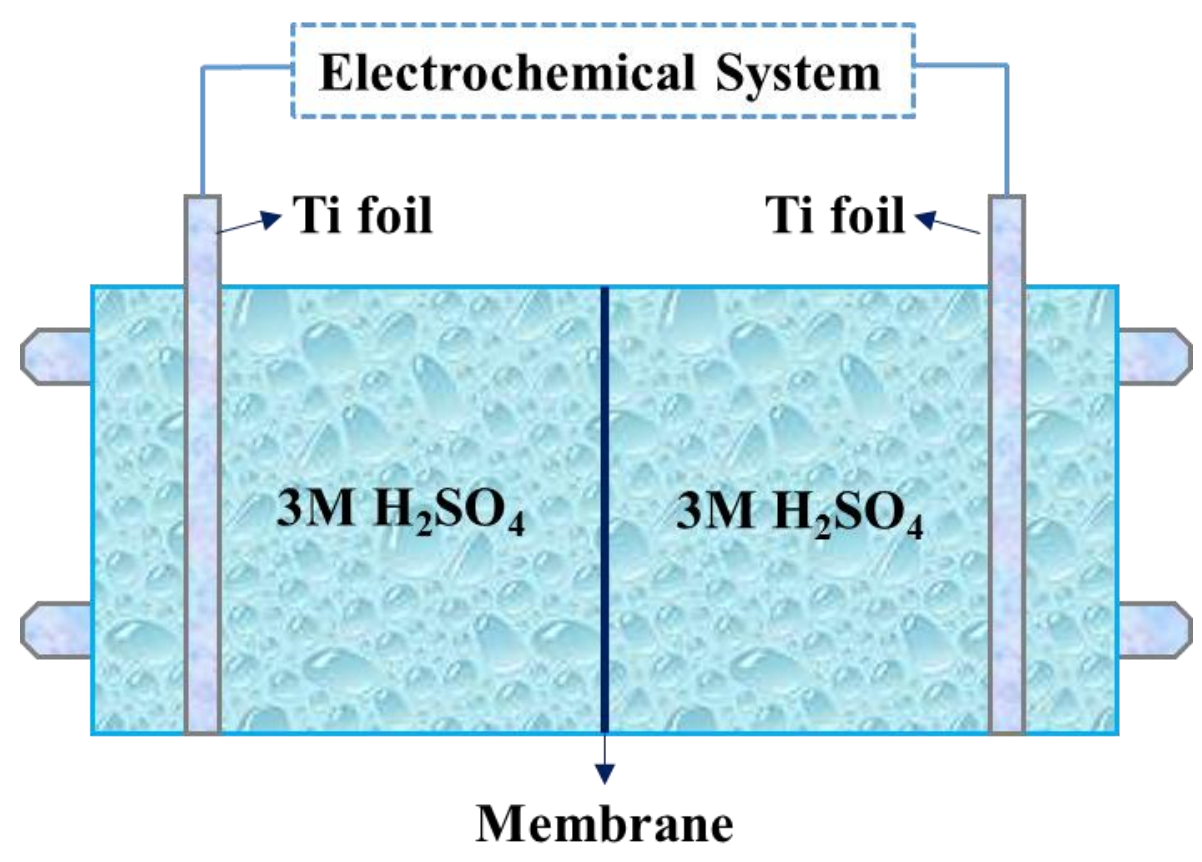

Figure S1. The conductivity cell for measuring area resistance. 


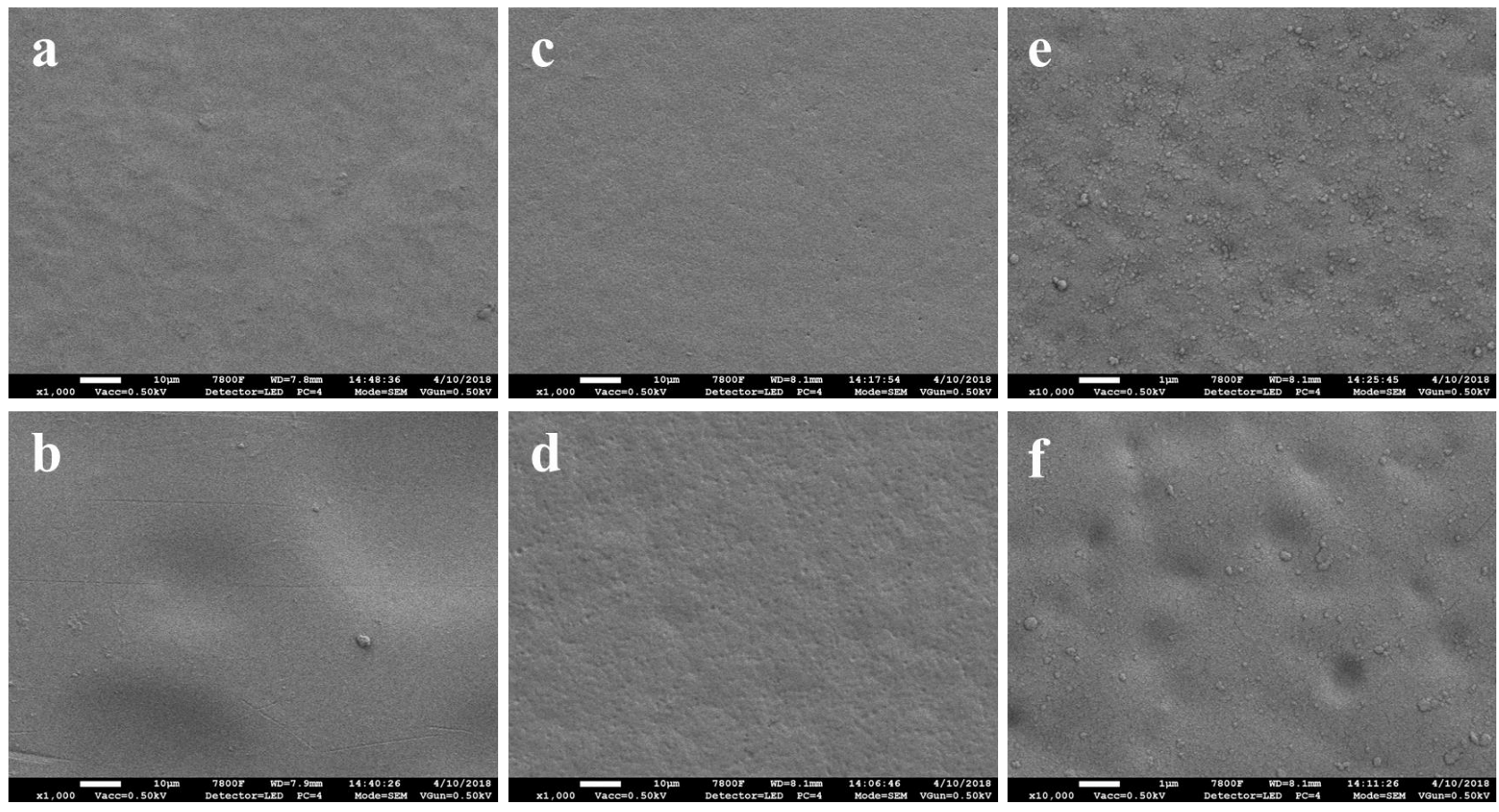

Figure S2. The surface SEM images of $\mathrm{NaCl}-\mathrm{XM}$ membranes. (a) $\mathrm{NaCl}-0 \mathrm{M}$ prepared in contact with nonsolvent (top), scale bar: $10 \mu \mathrm{m}$; (b) NaCl-0M prepared in contact with the glass plate (bottom), scale bar: $10 \mu \mathrm{m}$; (c) NaCl-5M (top), scale bar: $10 \mu \mathrm{m}$; (d) NaCl-5M (bottom), scale bar: $10 \mu \mathrm{m}$; (e) (f) magnified SEM images of (c) (d), respectively, scale bar: $1 \mu \mathrm{m}$. 


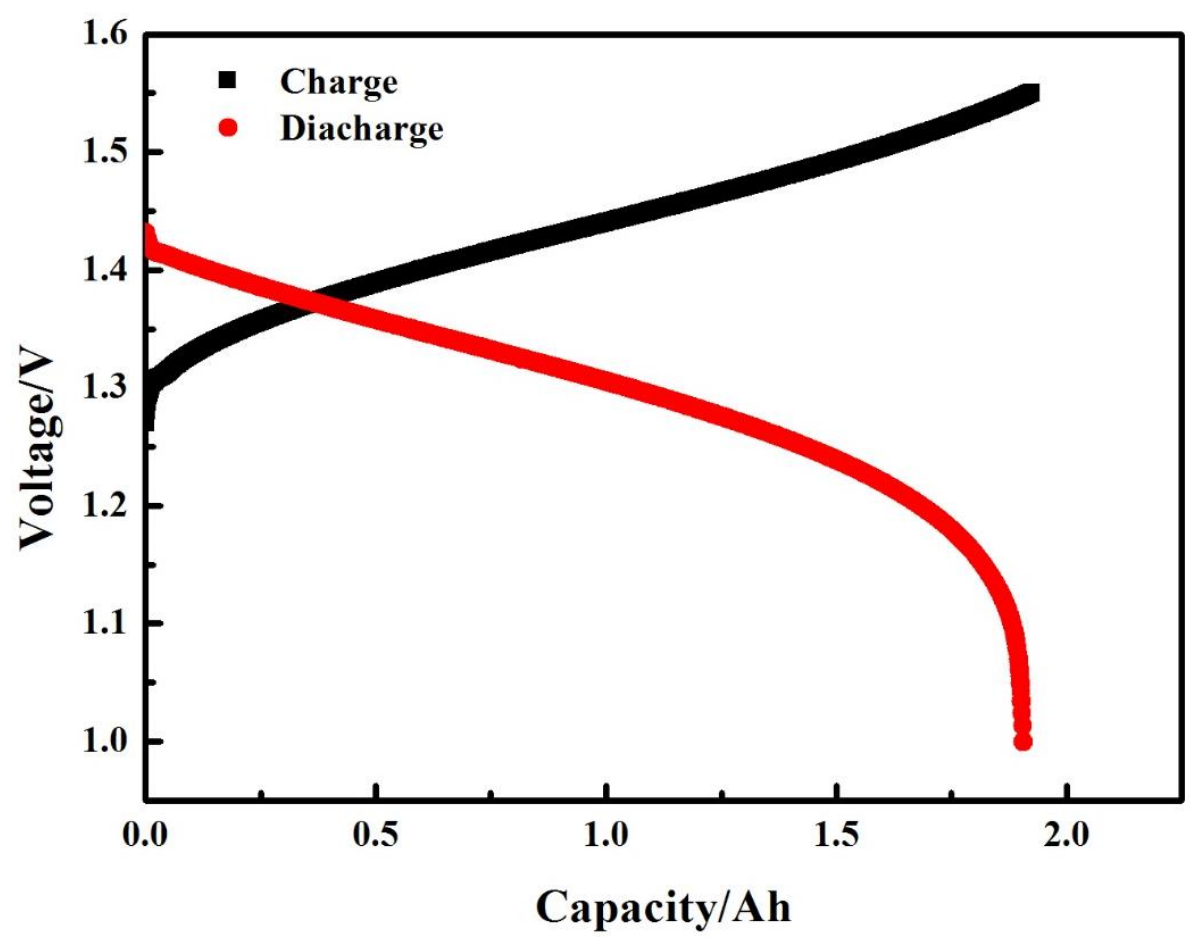

Figure S3. The galvanostatic charge-discharge curves of a VRB with $\mathrm{NaCl}-5 \mathrm{M}$ at the current densities of $80 \mathrm{~mA} \mathrm{~cm}^{-2}$. 


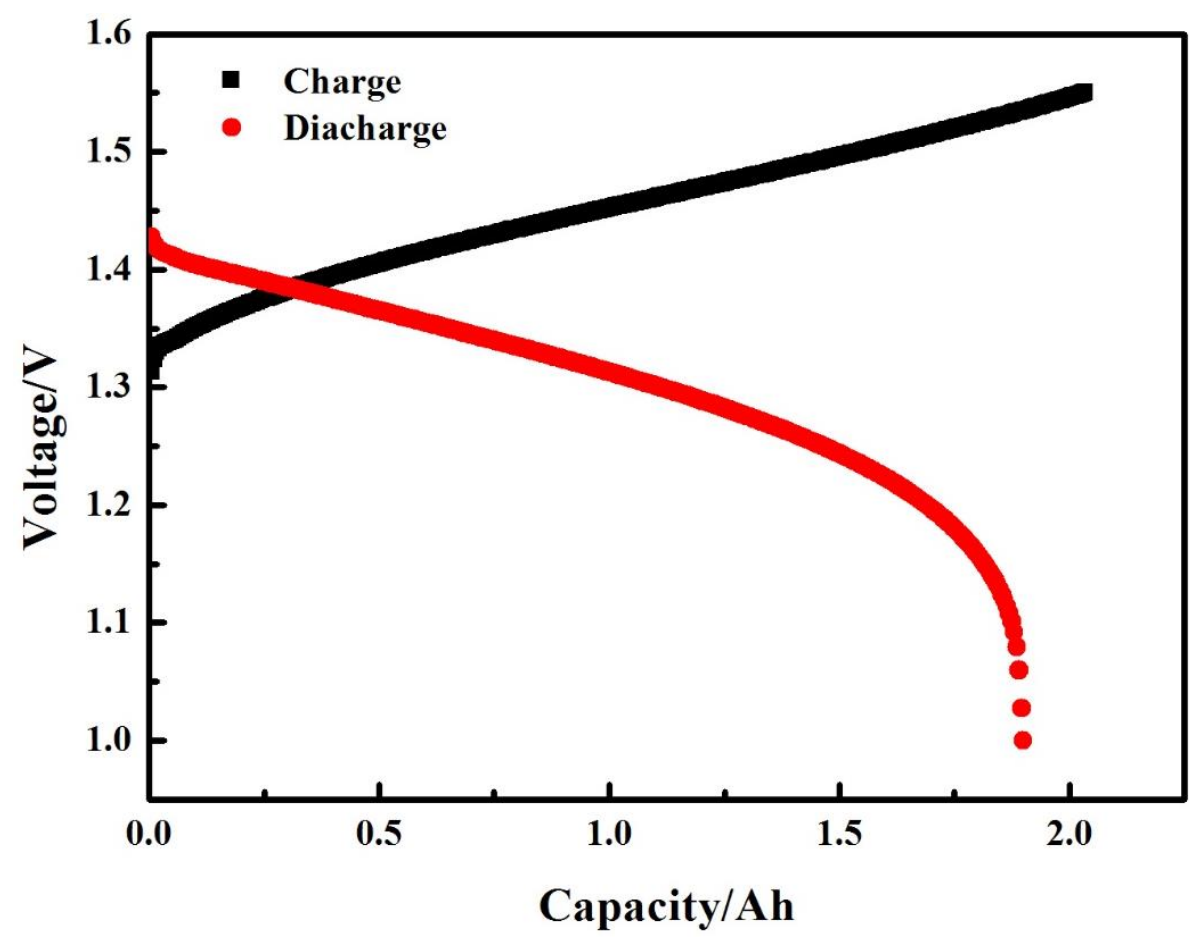

Figure S4. The galvanostatic charge-discharge curves of a VRB with Nafion 115 at the current densities of $80 \mathrm{~mA} \mathrm{~cm}^{-2}$. 


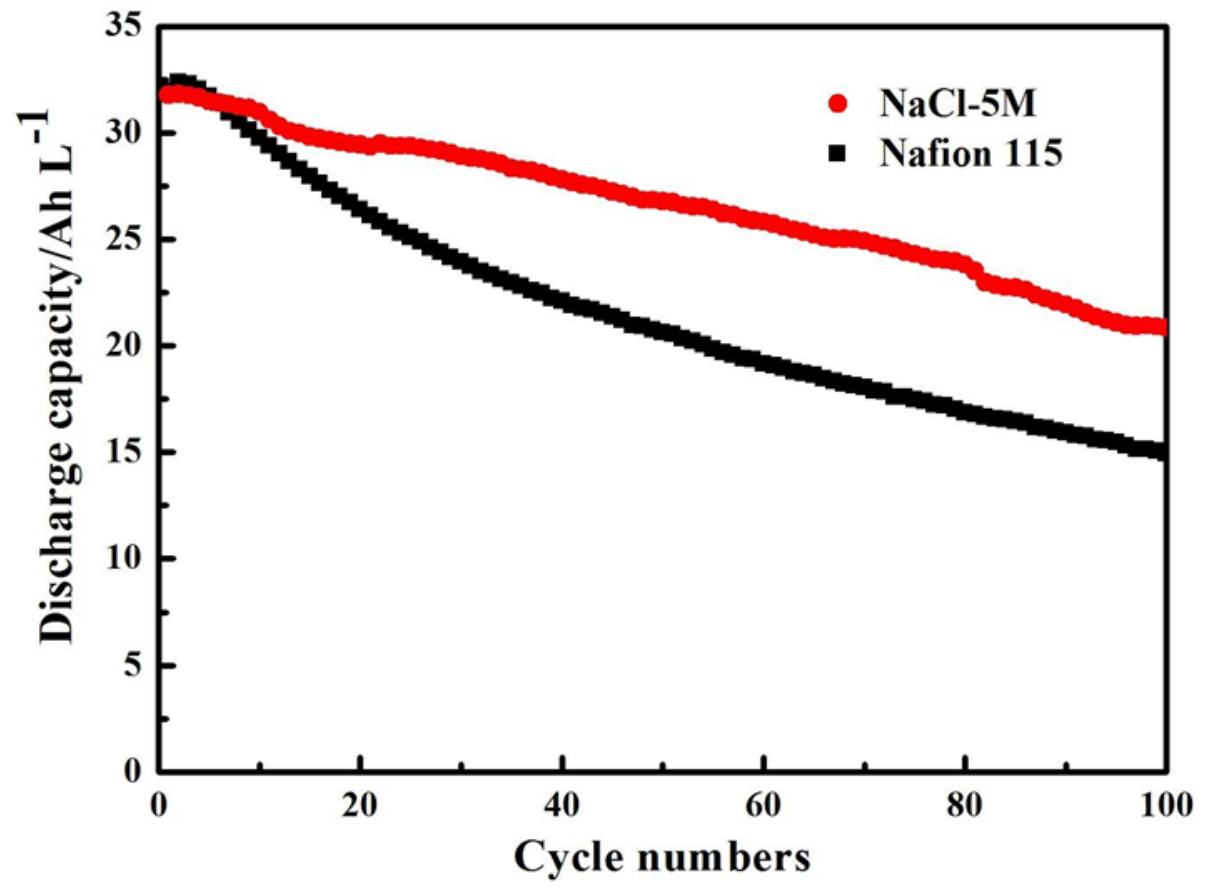

Figure S5. The capacity fading of VFBs with NaCl-5M and Nafion 115 over 100 cycles. 

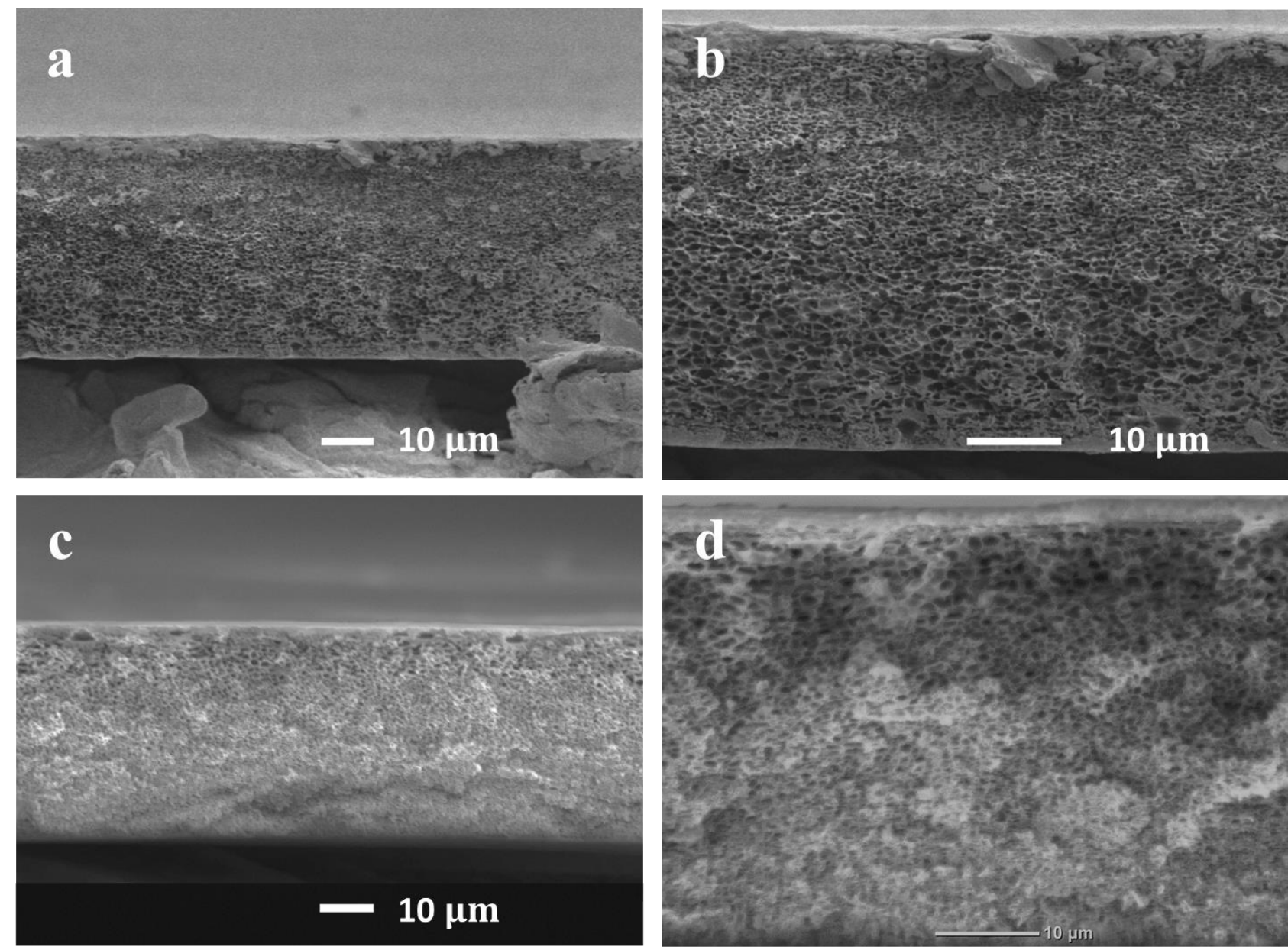

Figure S6. The cross-section SEM images of NaCl-5M before (a,b) and after (c,d) 10000 cycles. 

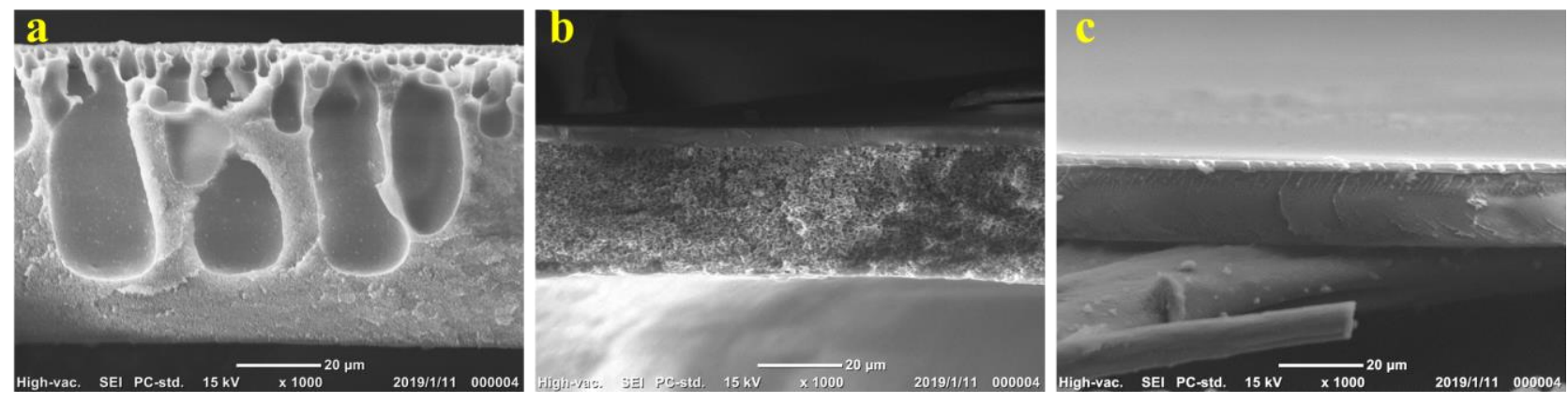

Figure S7. The cross-section SEM images of $\mathrm{MgCl}_{2}-\mathrm{XM}$ membranes at the same magnification. (a) $\mathrm{MgCl}_{2}-1 \mathrm{M}$; (b) $\mathrm{MgCl}_{2}-3 \mathrm{M}$; (c) $\mathrm{MgCl}_{2}-5 \mathrm{M}$. 